\title{
Internationales Register relevant für zukünftige Therapiestudien
}

Fragestellung: Wie ist der klinische Verlauf der Friedreich Ataxie (FRDA) einzuschätzen und welche Faktoren sind für die Planung randomisierter kontrollierter Therapiestudien von Bedeutung?

Hintergrund: Die rezessiv vererbte FRDA (GAA Triplet-Repeat Expansion) gehört zu den häufigsten erblichen Ataxien und führt in jungem Alter zu schwerer Beeinträchtigung mit eingeschränkter Lebenserwartung. Eine prospektive, longitudinale Studie mit einer größeren Kohorte zur Frage der Progression von Ataxie wie Nichtataxiesymptomen lag bislang nicht vor, um valide Parameter für den klinischen Verlauf und somit die Planung von Therapiestudien zu identifizieren.

Patienten und Methodik: In insgesamt elf europäischen Zentren wurden Patienten mit genetisch bestätigter FRDA eingeschlossen und dreimal im Jahresabstand und somit über einen Verlauf von zwei Jahren mit standardisierten, validierten Skalen untersucht. Als primärer Endpunkt wurde die SARA (Scale for the Assessment and Rating of Ataxia) mit einem Maximum von 40 Punkten gewählt. Da-

Reetz K, Dogan I, Hilgers RD et al. Progression characteristics of the European Friedreich's Ataxia Consortium for Translational Studies (EFACTS): a 2 year cohort study. Lancet Neurol 2016; 15: $1346-54$ bei geben höhere Werte schlechtere Befunde an. Sekundäre Endpunkte waren die INAS (Skalene Inventory of Non-Ataxia Signs), SCAFI (Spinocerebellar Ataxia Functional Index), PVF (Phonemic Verbal Fluency) und Skalen zur Alltagsaktivität und Lebensqualität (ADL, EQ5D-3L Index).

Die jährliche Progredienz wurde für jeden der Parameter unter Verwendung eines „linear mixed-effect modelling“ eingeschätzt. Im Weiteren wurden Effekte demografischer und krankheitsassoziierter Faktoren analysiert.

Ergebnisse: Von den anfänglichen 605 Patienten vervollständigten 506 das 1- und 474 das 2-Jahres-Follow-up. Die Rate der Progression im SARA lag in der gesamten Kohorte bei 0,77 \pm 0,06 Punkten pro Jahr. Die Verschlechterung im SARA war mit frühem Beginn der Erkrankung $(\leq 24$. Lebensjahr bei 505 der Patienten) und niedrigen SARA-Baseline-Scores assoziiert. Patienten mit mehr GAA-Triplet-Repeats wiesen eine deutlichere Progression im SARA-Score auf.

Auch für alle sekundären Outcome-Parameter ergab sich eine signifikante jährliche Verschlechterung.

Für die Detektion einer 50 \%igen Abnahme der Progression im SARA-Score in einer placebokontrollierten Therapiestudie über ein Jahr wurde eine notwendige Gruppengröße von 548 Patienten (274 pro Arm) ermittelt. Für einen 2-Jahres-Beobachtungszeitraum würde die notwendige Anzahl bei 184 Patienten und somit 92 pro Therapiearm liegen.

Schlussfolgerungen: Die longitudinale EFACTS-Studie legt dar, dass sich der SARA-Score für die Verlaufsbeurteilung von Ataxiesymptomen bei Patienten mit FRDA eignet und definiert notwendige Gruppengrößen für zukünftige Behandlungsstudien.

\section{- Kommentar von Marcus Gerwig, Essen}

\section{Bestätigung etablierter Ataxie-Scores}

Diese wichtige longitudinale Studie galt der Definition potenzieller Outcome-Variablen für zukünftige Therapiestudien bei der FRDA. Es konnte dargelegt werden, dass die SARA einen gut evaluierten klinischen Score für das Monitoring von Ataxiesymptomen im Zeitverlauf darstellt und im Vergleich zu anderen Skalen kompakter durchführbar ist. In Übereinstimmung mit früheren Studien ergab sich ein junges Alter zu Beginn der Erkrankung als wesentlicher Prädiktor für eine schnellere Progression. Ein prädiktiver Wert der GAA-RepeatLänge fand sich lediglich für die Gruppe mit höheren Repeats. Die Daten der Studie legen nahe, dass zwei Jahre einen kalkulierbaren Zeitraum für die Durchführung einer Therapiestudie bei der FRDA darstellen und weitere Selektionskriterien wie niedrige Baselinescores und jüngeres Alter bei Beginn der Erkrankung die notwendige Anzahl einzuschließender Patienten weiter senken könnten. Es wurden hier keine neurophy- siologischen oder bildgebenden Daten einbezogen. Die deutliche Änderung der ADL-Scores im Verlauf zeigt, dass die Berücksichtigung funktioneller Skalen in Studien bei Ataxien wichtig ist.

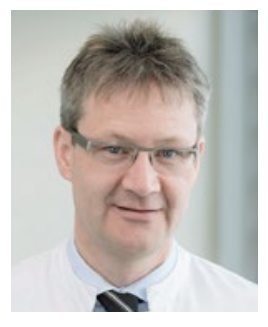

Prof. Dr. med. Marcus Gerwig, Essen

Klinik für Neurologie der

Universitätsklinik Essen

E-Mail: marcus.gerwig@uk-essen.de 